\title{
Wie können Bibliotheken Studierende fit für die Digital Humanities machen?
}

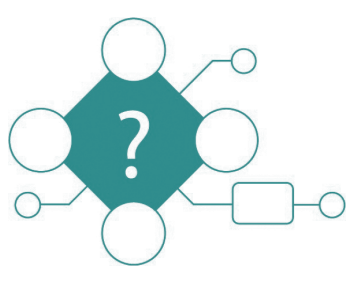

http://doi.org/10.1515/abitech-2018-3011

\begin{abstract}
Timo Glaser ist wissenschaftlicher Bibliothekar und arbeitet im Sachgebiet Forschungsnahe E-Dienstleistungen der Universitätsbibliothek Marburg. Er betreut die Bereiche Digitales Publizieren, Open Access, Forschungsinformationssysteme und hat das Digital Humanities Learning Lab entwickelt. Er beantwortet für $A B I$ Technik die aktuelle ABI Technikfrage: Wie können Bibliotheken Studierende fit für die Digital Humanities machen?
\end{abstract}

Bibliotheken agieren regelmäßig als Partnerinnen in Forschungsprojekten der digitalen Geisteswissenschaften. Besonders in den Bereichen Digitalisierung, Metadatenstandards, Forschungsdatenmanagement, digitales Publizieren und Langzeitarchivierung können Bibliothekarinnen und Bibliothekare mit ihren Kompetenzen Digital-Humanities-Projekte unterstützen.

Für Studierende gibt es allerdings kaum Angebote, Grundlagenkompetenzen im Bereich der Digital Humanities zu erwerben. Zwar gibt es zunehmend dedizierte Studiengänge und vereinzelt entsprechende Lehrveranstaltungen - für Studierende, Promovierende und auch Wissenschaftlerinnen und Wissenschaftler, die bislang keine Erfahrung in Digital Humanities haben und die keinen Zweitstudiengang Digital Humanities anstreben, sind die Möglichkeiten des Kompetenzerwerbs jedoch rar. Finden Praktiken der Digital Humanities in fachspezifischen Lehrveranstaltungen Anwendung, sind die Inhalte häufig aufgrund der konkreten Fragestellung kaum übertragbar oder alltagspraktisch nicht nachnutzbar (z. B. wenn in TEI und andere Metadatenschemata im Rahmen von Editionsprojekten eingeführt wird).

Bibliotheken können diese Leerstelle füllen, wenn sie die Forschungsorientierung im Bereich Digital Humanities aufbrechen und diese auch als Thema von „Informationskompetenz" sehen. Bibliotheken besitzen einschlägige Erfahrung darin, von curricularen Lehrveranstaltungen unabhängige, fach- und statusgruppenübergreifende Lernangebote $\mathrm{zu}$ entwickeln. So können Themen und Methoden vermittelt werden, die bereits im Studium oder in eigener Forschung, auch wenn es nicht explizite DigitalHumanities-Projekte sind, eingesetzt werden können, wie etwa „reguläre Ausdrücke“ und Auszeichnungssprachen, Digitalisierung und Texterkennung, Programmiersprachen und Datenmanagement.

Mit „regulären Ausdrücken“ lassen sich Zeichenketten abstrakt beschreiben. So können Texte nach Mustern durchsucht werden, um diese zu strukturieren, zu analysieren und auszuwerten. „Reguläre Ausdrücke“ ermöglichen dabei nicht nur einen ersten Einstieg in das Textund Datamining und das Konzept der Datenmodellierung, sondern können auch helfen, eigene Texte schnell zu überarbeiten. Sie bieten sich damit als Einstiegspunkt in die Digital Humanities an.

$\mathrm{R}$ und Python sind zwei Programmiersprachen, die sich für die Text- und Datenauswertung in den Digital Humanities zunehmend etabliert haben und recht einfach erlernt werden können. Beide können in sogenannten „Notebooks“ (in R-Studio bzw. Jupyter) eingesetzt werden und bieten dort die Möglichkeit, zusammen mit dem Code der Textanalyse die Ergebnisse in Form von Daten und Grafiken sowie die Erläuterungen und Interpretationen zu entwickeln und darzustellen. Damit eignen sie sich besonders für die etablierten Diskurspraktiken der Geisteswissenschaften und vermitteln die Grundlagen des „literarischen Programmierens“ und des „Data Storytelling“.

Durch solch niedrigschwellige und praxisbezogene Workshop-Angebote können Bibliotheken gerade nicht IT-affinen Geisteswissenschaftlerinnen und Geisteswissenschaftlern Zugänge ermöglichen, Digital-HumanitiesThemen „entzaubern“, auf Hilfsmittel zum eigenständigen Weiterlernen verweisen und Beratungspunkte anbieten, wenn diese in einem while-Loop hängenbleiben.

\section{Autoreninformationen}

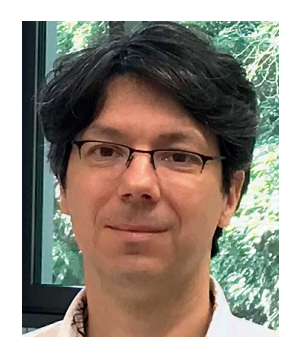

Philipps University Marburg

University Library

Deutschhausstraße 9

35032 Marburg

orcid.org/0000-0002-2590-647X 